\title{
Use of ROTEM and MEA in a cardiac surgical patient with ITP
}

\author{
Junko Ichikawa $\cdot$ Mitsuharu Kodaka $\cdot$ \\ Goro Kaneko
}

Received: 3 May 2013/Accepted: 23 July 2013/Published online: 21 August 2013

(C) Japanese Society of Anesthesiologists 2013

Keywords Idiopathic thrombocytopenic purpura . ROTEM $\cdot$ MEA

To the Editor:

Perioperative management of idiopathic thrombocytopenic purpura (ITP) is challenging because the platelet count does not directly indicate platelet function. We report the use of rotation thromboelastometry (ROTEM; Tem International, Munich, Germany) and multiple electrode aggregometry (MEA) using a Multiplate analyzer (Dynabyte Medical, Munich, Germany) (Supplemental Fig. 1) to manage a 71-year-old man with an 8-year history of ITP who required an aortic valve replacement. ITP is characterized by the formation of antiplatelet antibodies that target platelet membrane glycoproteins, resulting in accelerated platelet destruction. After induction of anesthesia, the maximum clot firmness (MCF) was low in the EXTEM test but was normal in the FIBTEM test, and MEA measurements were all below the normal range, which are independent of activators (Supplemental Tables 1, 2). These results indicated a reduction in the platelet component as reflected by MCFextem-MCFfibtem [1] and slower and less dense occupation of the sensor wires on MEA, decreasing the aggregation velocity and the maximum aggregation [2]. During cardiopulmonary bypass, the MCF

Electronic supplementary material The online version of this article (doi:10.1007/s00540-013-1690-9) contains supplementary material, which is available to authorized users.

J. Ichikawa $(\bowtie) \cdot M$. Kodaka · G. Kaneko

Department of Anesthesiology, Tokyo Women's Medical

University Medical Center East, 2-1-10 Nishiogu,

Arakawa-ku, Tokyo 116-8567, Japan

e-mail: htwfx872@yahoo.co.jp was reduced in EXTEM and FIBTEM tests, and these results together with all MEA measurements indicated hemodilution, coagulation factor depletion, and platelet dysfunction (Supplemental Tables 1,2). According to the ROTEM-based algorithm, $30 \mathrm{U}$ platelets and $5 \mathrm{U}$ fresh frozen plasma were transfused, which normalized the ROTEM data and the unsatisfactorily corrected MEA data (Supplemental Tables 1,2). These results reflected reduced platelet aggregation because of apheresis and storage. MEA has been reported to be useful for assessment of platelet responsiveness to antiplatelet therapy. As ROTEM is unable to evaluate primary hemostasis, use of ROTEM and MEA together might be functionally complementary. However, the patient did not require any more blood products, and the results of MEA did not affect the transfusion decision.

\section{References}

1. Gunduz E, Akay OM, Bal C, Gulbas Z. Can thrombelastography be a new tool to assess bleeding risk in patients with idiopathic thrombocytopenic purpura? Platelets. 2011;22:516-20.

2. Hanke AA, Roberg K, Monaca E, Sellmann T, Weber CF, RaheMeyer N, Görlinger K. Impact of platelet count on results obtained from multiple electrode platelet aggregometry (Multiplate). Eur J Med Res. 2010;15:214-9. 